\title{
A Rare Case of Vanishing Lung Syndrome
}

\author{
Venkategowda $\mathrm{PM}^{1^{*}}$ and $\mathrm{SM} \mathrm{Rao^{2 }}$ \\ ${ }^{1}$ Department of Critical Care Medicine, Apollo Hospital, Bangloor, India \\ ${ }^{2}$ Deartment of Critical Care Medicine, Yashoda Multi-Speciality Hospital Somajiguda, Hyderabad, India
}

${ }^{*}$ Corresponding author: Venkategowda PM, Department of Critical Care Medicine, Apollo Hospital, Sheshadripuram, Bengaluru 560020. India. Mobile No: 08897575704 E-mail: drpradeepmarur@gmail.com

Citation: Venkategowda PM (2017) A Rare Case of Vanishing Lung Syndrome. SAJ Case Reports 4: 103

Article history: Received: 23 December 2016, Accepted: 24 January 2017, Published: 26 January 2017

Keywords: Vanishing lung; Emphysema; Pneumothorax; Bullectomy

Learning Objective: VLS: Vanishing Lung Syndrome; HRCT: High Resolution Computed Tomography; ICD: Intercostal Drainage; ER:

Emergency Room

Sir,

Vanishing lung syndrome (VLS) or idiopathic giant bullous emphysema is a rare condition characterized by giant bulla in one or both upper lobes of the lung, occupying at least one third of hemithorax. The lung appears to be disappearing on routine chest $\mathrm{X}$-ray and it is often mistaken as pneumothorax. Routine chest X-ray cannot differentiate between pneumothorax and giant bulla, HRCT chest is the investigation of choice. The placement of intercostal drainage (ICD) with suspicious of pneumothorax can cause iatrogenic pneumothorax, hemothorax or death.

A 36 year old male without any comorbids, non smoker and non alcoholic came to our emergency room (ER) with history of road traffic accident (hit by a two wheeler) 2 hours back. On examination, patient was conscious and coherent with spo2- $94 \%$ on room air (100\% with face mask- 6 L oxygen) and respiratory rate of 24 breaths / minute. On auscultation of chest, decreased air entry along the right basal region with hyper resonant note on percussion was noted. There was a definitive deformity of left arm. Thansthorasic 2D Echo was done which showed normal left ventricular function without any regional wall motion abnormality but with mild rise in pulmonary artery pressure. Chest radiograph (AP VIEW) showed hyperlucent right basal region of lung (Figure 1). Alpha-1-antitrypsin levels were reduced. Pulmonary function test was not done (patient was not willing for further test in view of financial constraints). Initially thought it may be pneumothorax and hence computed tomography of lung (Figure 2 and Figure 3) was done which confirmed giant bullous emphysema (vanishing lung syndrome). Left arm X-ray showed fractured shaft of humerus for which plating was done. Our patient refused for bullectomy which is the treatment of choice for VLS (but agreed for regular follow up).

Vanishing lung syndrome also known as Primary bullous disease of the lung was first described by Burke in 1937 [1]. Robert's and colleagues proposed radiological criteria [2] which include giant bulla in one or both upper lobes, occupying at least one third of hemithorax and compression of surrounding normal lung parenchyma. Giant bullae are sometimes seen at bases [3].

Risk factors include Smoking [4], Alpha 1 antitrypsin deficiency, Marijuana abuse [5] and connective tissue diseases. Vanishing lung syndrome (VLS) seen commonly in young males [6] and most of them are asymptomatic and usually diagnosed accidentally during routine chest $\mathrm{X}$-ray. This is a progressive condition and patients usually present with worsening dyspnoea or severe acute breathlessness in case of spontaneous rupture of bulla. Definitive investigation is HRCT Chest [7]. Treatment is mainly surgical. Limited bullectomy has shown some good outcome [8]. The presence of VLS in young non smoker and involvement of basal part of the lung is a rare phenomenon which was seen in our patient. High suspicious of Vanishing lung syndrome along with bullectomy and quitting of smoking can reduce morbidity and mortality.

\section{Acknowledgments}

We gratefully acknowledge management of the hospital for their valuable support. 


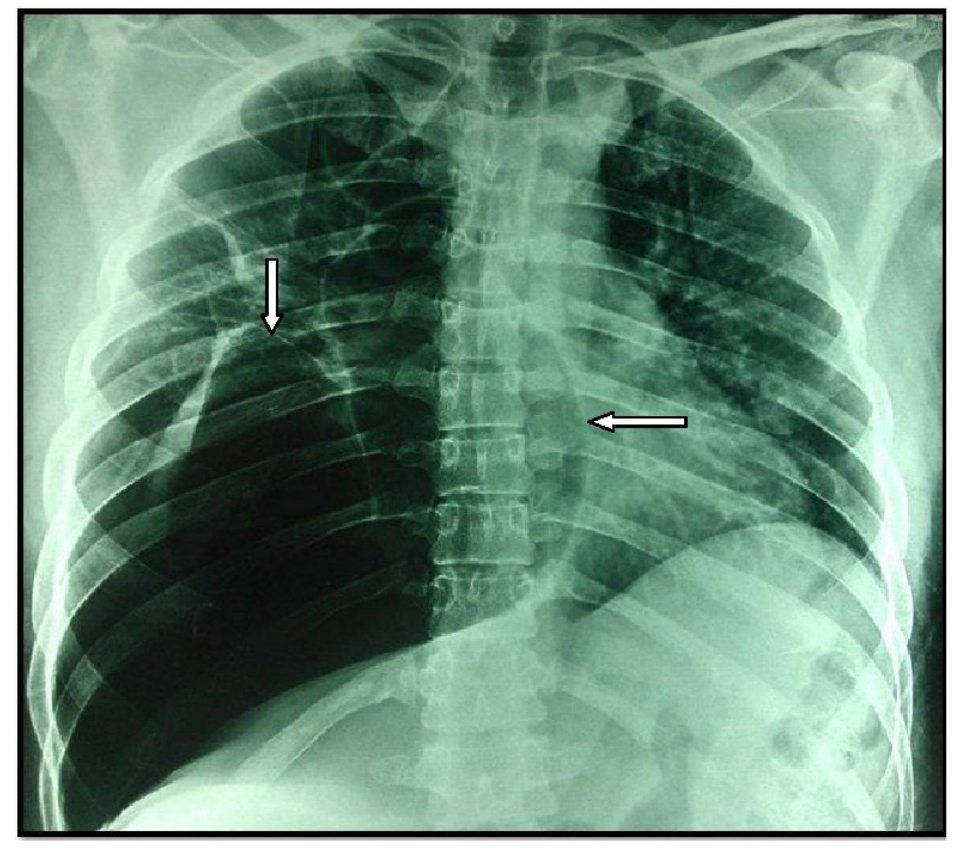

Figure 1: Chest X-ray (AP VIEW) showing giant bulla at right lower lobe

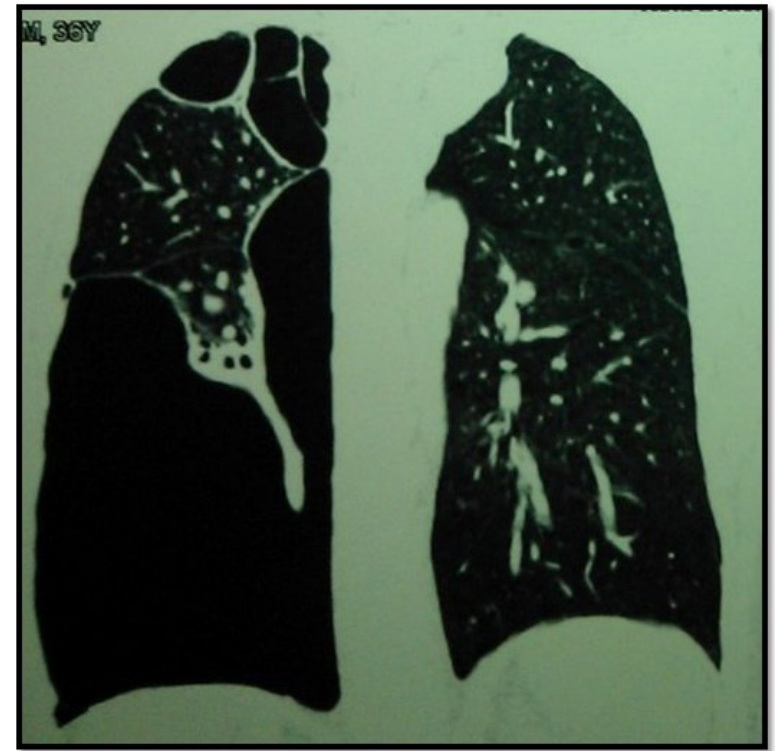

Figure 2: CT chest (Longitudinal view) confirming right lower lobe giant bulla

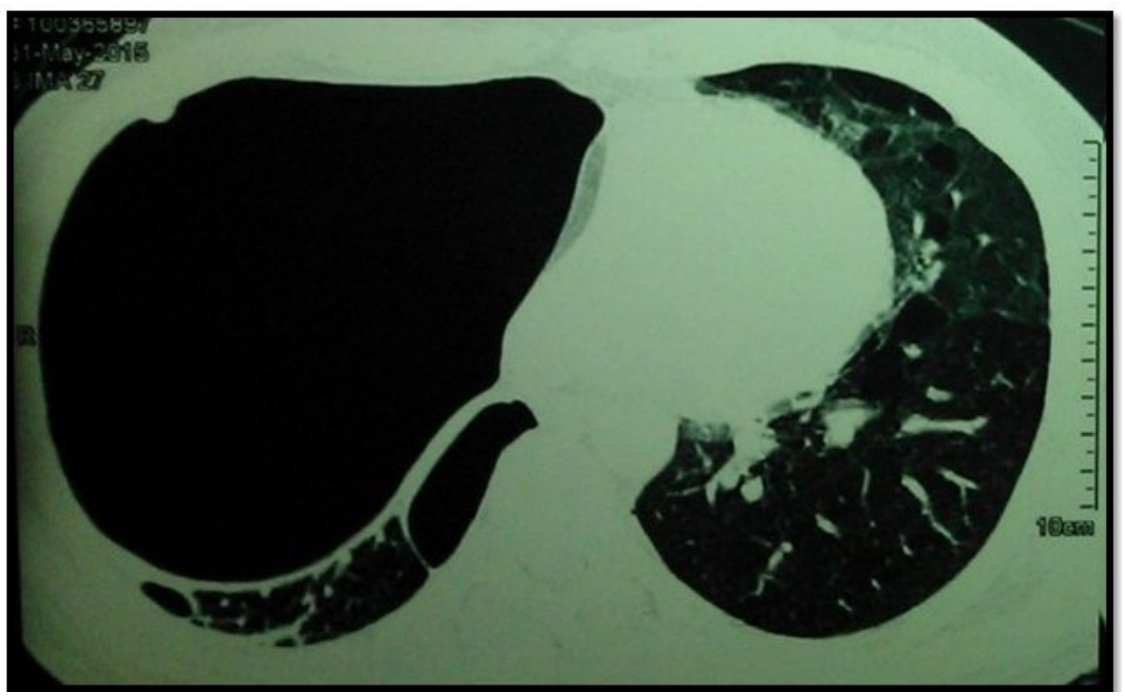

Figure 3: CT chest (Transverse view) confirming right sided giant bulla 


\section{References}

1. Burke R (1937) Vanishing lungs: a case report of bullous emphysema. Radiology 28: 367-71.

2. Roberts L, Putman CE (1987) Vanishing lung syndrome: upper lobe bullous pneumopathy. Revista Interamericana de Radiologia 12: 249-55.

3. Basheer Tashtoush, Fernando GI, Roya M, Anas H, Laurence S., et al. (2014) Vanishing Lung Syndrome in a Patient with HIV Infection and Heavy Marijuana Use. Case Rep Pulmonol : 285208

4. Ladizinski B, Sankey C (2014) Vanishing Lung Syndrome. Can Respir J 370: e14.

5. Sood N, Sood N (2011) A Rare Case of Vanishing Lung Syndrome. Case Rep Pulmonol 957463, 1155/957463.

6. Laleng MD, Hajong R, Das R, Topno N (2010) Vanishing lung syndrome. Indian J Surg 72: 75-6.

7. Morgan MDL, Strickland B (1984) Computed tomography in the assessment of bullous lung disease. Br J Dis Chest $78: 10-25$.

8. Palla A, Desideri M, Rossi G (2005) Elective surgery for giant bullous emphysema: a 5-year clinical and functional follow-up. Chest 128: 2043-50. 